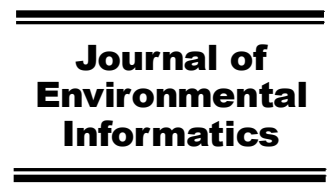

www.iseis.org/jei

\title{
Data Driven Models as A Powerful Tool to Simulate Emerging Bioprocesses: An Artificial Neural Network Model to Describe Methanotrophic Microbial Activity
}

\author{
A. AlSayed ${ }^{1}$, M. Soliman ${ }^{1}$, R. Shakir ${ }^{1}$, E. Snieder ${ }^{1}$, A. Eldyasti $^{1}$, and U. T. Khan ${ }^{1}{ }^{*}$ \\ ${ }^{1}$ Department of Civil Engineering, Lassonde School of Engineering, York University, Toronto, ON M3J 1P3, Canada
}

Received 9 September 2019; revised 19 May 2020; accepted 26 May 2020; published online 25 March 2021

\begin{abstract}
The vision for sewage treatment plants is being revised and they are no longer considered as pollutant removing facilities but rather as water resources recovery facilities (WRRFs). However, the newly adopted bioprocesses in WRRFs are not fully understood from the microbiological and kinetic perspectives. Thus, large variations in the outputs of the kinetics-based numerical models are evident. In this research, data driven models (DDM) are proposed as a robust alternative towards modelling emerging bioprocesses. Methanotrophs are multi-use bacterium that can play key role in revalorizing the biogas in WRRFs, and thus, a Multi-Layer Perceptron Artificial Neural Network (ANN) model was developed and optimized to simulate the cultivation of mixed methanotrophic culture considering multiple environmental conditions. The influence of the input variables on the outputs was assessed through developing and analyzing several different ANN model configurations. The constructed ANN models demonstrate that the indirect and complex relationships between the inputs and outputs can be accurately considered prior to the full understanding of the physical or mathematical processes. Furthermore, it was found that ANN models can be used to better understand and rank the influence of different input variables (i.e., the physical parameters that influence methanotrophs) on the microbial activity. Methanotrophic-based bioprocesses are complex due to the interactions between the gaseous, liquid and solid phases. Yet, for the first time, this study successfully utilized DDM to model methanotrophic-based bioprocesses. The findings of this research suggest that DDM are a powerful, alternative modeling tool that can be used to model emerging bioprocesses towards their implementation in WRRFs.
\end{abstract}

Keywords: artificial neural network (ANN), data driven models (DDM); kinetics, kinetics-based models (KBM), modelling, methanotrophs, upscaling, water resources recovery facilities (WRRFs)

\section{Introduction}

Recently, there has been a focus to reconsider the vision for sewage treatment plants (STPs) (McCarty et al., 2011). This focus is due to the fact that STPs consume enormous amount of energy, typically based on fossil fuels. For instance, in North America around $4 \%$ of the annual electrical energy consumption is used for the operation of STPs and this fossil fuel-based energy is responsible for emitting 45 million tons of carbon dioxide equivalents, representing $75 \%$ of total greenhouse gases emissions from STPs (Canadian Biogas Association, 2013; Liao et al., 2016; US EPA, 2016). This significant amount of energy consumption and greenhouse gas emission is not only of environmental concern but also of economic interest (Asztalos and Kim, 2017), since it has been suggested that the potential energy contained in sewage is nearly 5 times higher than the energy consumed during the treatment process (Gu et al., 2017; Puyol et al., 2017). Hence, STPs should no longer be considered as pollutant removing facilities, but as facilities that couple the sewage treatment process with the generation of energy

${ }^{*}$ Corresponding author. Tel.: 416.736 .2100 ext. 55890

E-mail address: usman.khan@lassonde.yorku.ca (U. T. Khan).

ISSN: 1726-2135 print/1684-8799 online

(C) 2021 ISEIS All rights reserved. doi:10.3808/jei.202000446. and value-added products, which are known as water resources recovery facilities (WRRFs). Therefore, more attention to alternative and novel bioprocesses is needed to support the novel WRRFs processes and replace the conventional activated sludge-based processes (Wan et al., 2016). However, most of the suggested novel processes in the literature have not yet been fully understood from both the microbiological and kinetic perspectives (Puyol et al., 2017). Consequently, the development of reliable models that can describe their microbial activity is a crucial milestone towards converting conventional STPs into WRRFs.

Carbon redirection towards anaerobic digestion (AD) is a key strategy to consolidate the WRRFs concept (Jimenez et al., 2015). It is advantageous due to its applicability in relatively low temperatures and the production of easily digestible sludge (Sancho et al., 2019). AD is a widely adopted technology known for its capacity for sludge minimization under low energy requirements since no aeration is required, whereas, biogas is released as the value-added product (Tchobanoglous et al., 2003; Chen et al., 2008). Nevertheless, multiple obstacles hinder the direct utilization of the produced biogas such as the presence of impurities and its low handling and collecting capabilities (Ge et al., 2014).

Methane oxidizing bacteria (methanotrophs) have the ex- 
clusive ability to directly utilize the $\mathrm{AD}$ driven biogas and revalorize it into higher value-added products like single cell proteins, methanol, and biopolymers (Fergala et al., 2018a). Unlike the other types, type I methanotrophs have higher growth rates and energy efficiency, which makes it more attractive for industrial applications (AlSayed et al., 2018a). Thus, the development of a model that can describe type I methanotrophs microbial activity would be beneficial as a vital step to be up scaled and adopted in WRRFs. Unfortunately, type I methanotrophs formed cultures are more sensitive than the other types of methanotrophic bacteria to the fluctuation of the surrounding environmental conditions. The factors that can affect the methanotrophic bacteria include nitrogen concentration, copper concentration, substrate concentration, and biomass density (AlSayed et al., 2018a). Thus, the effects of these factors on type I methanotrophs activity should be considered to appropriately predict and design any methanotrophic based numerical models and bioreactors.

\subsection{Background of Kinetics-Based Models}

Generally speaking, numerical models may be divided into two categories: (i) physical or physics-based models, which depend on the full understanding of the process behavior and principles (Soliman and Eldyasti, 2017); and (ii) data driven models (DDM) which rely on empirical mathematical equations assessed from the analysis of input and output (or target) parameters rather than the explicit underlying physical processes (Solomatine and Ostfeld, 2008). Kinetics-based models (KBM), a type of physical based models, have been extensively studied to describe the methanotrophic microbial activity (Delhoménie et al., 2008; Rostkowski et al., 2013; Ménard et al., 2014; Ordaz et al., 2014). In order to develop any microbial $\mathrm{KBM}$, five coefficients must be experimentally attained: maximum specific growth rate $\left(\mu_{\max }\right.$, day $\left.^{-1}\right)$, Monod half saturation constant $\left(K_{s}, \mathrm{mg}_{\text {substrate }} / \mathrm{L}\right)$, maximum specific substrate (methane) utilization rate $\left(q_{\max }, \mathrm{mg}_{\text {substrate }} \cdot \mathrm{mg}_{\text {cells }}{ }^{-1}\right.$.day $\left.{ }^{-1}\right)$, the true growth yield ( $\left.Y_{\text {true }}, \mathrm{mg}_{\text {cells }} / \mathrm{mg}_{\text {substrate }}\right)$ and the endogenous decay rate $\left(K_{d}\right.$, day $\left.^{-1}\right)$. Thereafter, the specific growth rate $\left(\mu\right.$, day $\left.^{-1}\right)$, the specific methane utilization rate $\left(q, \mathrm{mg}_{\text {substrate }} \cdot \mathrm{mg}_{\text {cells }}{ }^{-1}\right.$.day $\left.{ }^{-1}\right)$, the actual substrate uptake rate $\left(M U R_{\text {actual }}, \mathrm{mg}_{\text {substrate }} /\right.$ day), and the apparent yield $\left(Y_{\text {app }}, \mathrm{mg}_{\text {cells }} / \mathrm{mg}_{\text {substrate }}\right)$ can be calculated using Equations 1 to 4 (Rittmann and McCarty, 2012). The calculated parameters are commonly used to describe any microbiological system performance:

$$
\begin{aligned}
& \mu=\mu_{\max } \frac{\text { substrate conc. }}{K_{s}+\text { substrate conc. }}=\mu_{\max } \frac{S}{K_{s}+S} \\
& q=q_{\max } \frac{S}{K_{s}+S} \\
& M U R=\frac{-\Delta S}{\Delta t}=\text { biomass conc. } q_{\max } \frac{S}{K_{s}+S}=X_{a} \cdot q \\
& Y_{\text {app. }}=Y_{\text {true }}-\left(K_{d} / q\right)
\end{aligned}
$$

Unfortunately, the reported values for the KBM coefficients have a very wide range (Rostkowski et al., 2013) leading to highly uncertain model predictions. The reported $\mu_{\max }$ varies from 0.43 to 8.16 day $^{-1}$ which results in notably different prediction of $\mu$. As a result, the highest predicted value can be 19 times higher than the lowest predicted value (Heijnen and Roels, 1981; Boiesen et al., 1993; Delhoménie et al., 2008; Rostkowski et al., 2013; Rostkowski et al., 2013; Ménard et al., 2014; Ordaz et al., 2014). Furthermore, Ordaz et al. (2014) has recently reported $K_{s}$ up to 100 times lower than other values within the literature, again showing the significant variance in the reported values (Delhoménie et al., 2008; Ménard et al., 2014; Ordaz et al., 2014). Similarly high variability has been reported for growth yields: growth yields of $0.585 \mathrm{mg}_{\text {cells }} / \mathrm{mg}_{\mathrm{CH} 4}$ were obtained using type I methanotrophs pure culture of $\mathrm{Me}$ thylococcus capsulatus (Siniscalchi et al., 2015), while a new type I methanotrophs isolated from solid state anaerobic digesters had a growth yietd of $0.19 \mathrm{mg}_{\text {cells }} / \mathrm{mg}_{\mathrm{CH} 4}$ (Sheets et al., 2016). Most of the reported yields are apparent yield ( $\left.Y_{\text {app. }}\right)$ and not the true yield which can be used in bioreactor design parameters. In other words, the calculated yield is expected to vary from all the reported values at different conditions. To the best of our knowledge, very limited studies have reported the $K_{d}$ and also showed notable differences (Boiesen et al., 1993). Regarding the $q_{\max }$, few values were reported within the literature rang-

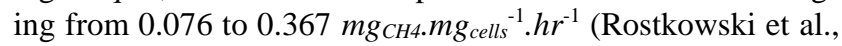
2013; López et al., 2014; Ordaz et al., 2014). These studies demonstrate that KBM coefficient values vary widely in the reported literature, making it difficult to assess the accuracy and utility of KBM type models. Figure 1 shows the probability density function of the specific growth rate $(\mu)$ using the values of $\mu_{\max }$ and $K_{s}$ as reported in the literature. It illustrates the notable variation in the KBM coefficients which results in a large difference in the expected performance and design parameters with $\mu$ as an example. Additionally, the experimentally determined KBM coefficients do not consider the factors that have been reported to affect the methanotrophic activity (e.g., the biomass density, copper and nitrogen concentrations). As a result, a wide range of kinetics have been reported in literature, since the experiments conducted to obtain these KBM coefficients have not been performed under the same experimental conditions (Rostkowski et al., 2013; Ordaz et al., 2014). Even though multiple correction equations have been developed to consider some of the factors (e.g., the temperature and the substrate concentration), most of the other factors that influence methanotrophic activity have not been successfully modelled yet.

\subsection{Data-Driven Models for Simulating Methanotrophic Culture}

DDM have the potential to simulate any biological or methanotrophic culture performance whilst considering all the needed factors that may affect the culture performance. Interestingly, these factors can be considered and simulated in a DDM, even though, the impact of these conditions are not fully understood or can be mathematically described (Khan and Valeo, 2016a, 2017a, 2017b). In other words, DDMs allow to model a system 


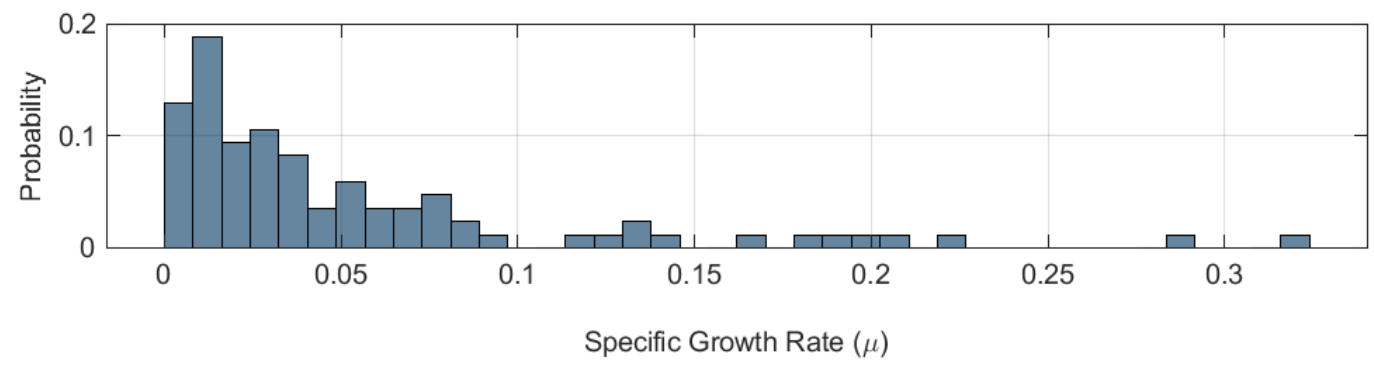

Figure 1. The probability density function of the specific growth rate using the values of $\mu_{\max }$ and $K_{s}$ as reported in the literature.

based on the available input data (e.g., the KBM coefficients) and the required output data (e.g., methanotrophic biomass). Figure 2 shows the framework for both the KBM and the DDM models. It is noteworthy that the experiments performed to obtain the data used for developing the DDM model should be well designed to achieve two objectives. The first objective is to consider all the factors that may affect the simulated process performance (Maier and Dandy, 2000). For instance, specific nutrients directly affect some enzymes activity like the copper effect on the expression of the methane monooxygenase (MMO) enzyme (Semrau et al., 2010) or the competitive inhibitory effect between the ammonium and the methane on the ammonia monooxygenase (AMO) and MMO enzymes must be considered (Soliman and Eldyasti, 2016; AlSayed et al., 2018a). Secondly, the experimental parameters should be well distributed over all the realistic and viable values of the parameters of interest (Maier and Dandy, 2000a). For example, wide range of all the possible nitrogen concentrations should be used in the experiments to fully represent the nitrogen effect on the culture performance. Collectively, it can be concluded that a DDM based on data obtained from well-designed experiments may be used to predict type I methanotrophs culture in order to design methanotrophic cultivation bioreactors.

One common and increasingly popular type of DDM are artificial neural networks (ANN) which have been widely used for modeling complex relationships between inputs and outputs in nonlinear systems in several engineering disciplines (Nourani et al., 2012; Kisi et al., 2013; Khan and Valeo, 2016b). The Multi-Layer Perceptron (MLP) model is a type of ANN, and consists of one input layer, at least one hidden layer and one output layer. These layers have processing elements called neurons that are interconnected through connection links; each link has a weight and bias that define the strength of the connection (Govindaraju, 2000). ANNs have several advantages such as the ability of detecting complex non-linear relationship between dependent and independent variables. However, ANN has some challenges such as the requirement of high computational power (for very large datasets), the inability of extrapolating result beyond the range of data used for training, and challenges arising from the lack of the uncertainty analysis which might impact the results of the models.

In this research, a novel proof-of-concept modelling approach is proposed to demonstrate that DDM can be used to efficiently model bioprocesses in WRRFs, instead of the more common KBM approach. Specifically, an innovative approach is proposed to develop and optimize a series of MLP ANN to simulate the cultivation of mixed culture methanotrophs type I bacteria enriched from sewage sludge considering multiple operational and/or environmental conditions. The proposed models are developed for a wide range of input parameter values and operational conditions which is not possible to do under the standard KBM approach. The significance of the proposed approach is twofold: first, the ability of DDMs (specifically ANNs) to model complex bioprocesses is demonstrated, and second, the ANNs are used to highlight the relative importance or influence of input parameters on the bioprocesses. Results from this research will help improve the designs of methanotrophic cultivation bioreactors, give a better understanding of the important variables controlling the methanotrophic processes, and can ultimately lead to higher resource recovery in WRRFs. Such an approach can facilitate the adoption of novel technologies and develop better understanding of the effect of the operational conditions within WRRFs.

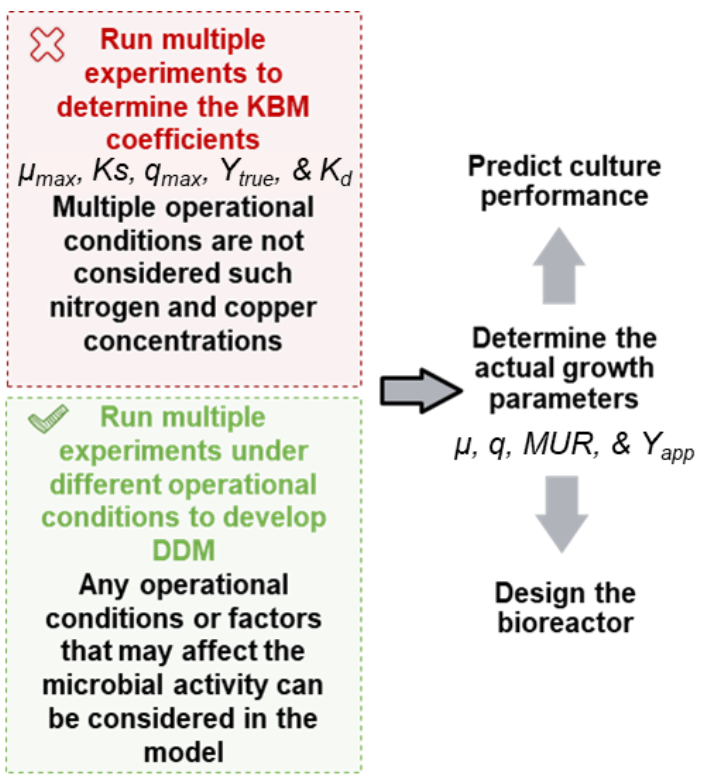

Figure 2. KBM and DDM framework to predict culture performance and design the bioreactor.

\section{Methods}

To develop and analyze the ANN models, first, five "core" 
and four "ratio" factors are used as inputs to predict methanotrophic biomass density and methane concentration (the factors needed to determine the KBM coefficients). The impact and influence of these factors on ANN model performance is then analyzed. Following this, input variable selection (IVS), a key component in ANN model development and uncertainty quantification (Ramesan et al., 2018, Snieder et al., 2020), is performed to determine the most influential "core" factors to further refine the ANN model. Lastly, the architecture of the ANN is then optimized by investigating the impact of the number of nodes (neurons) and the amount of data used for training the model. The following sections provide an overview of the methods used to collect the data, develop the ANN models, and to optimize the ANN architecture.

\subsection{Data Collection}

The data were collected from multiple batch experiments performed over 6 months using methanotrophic culture enriched from sewage sludge obtained from the Humber Wastewater Treatment Plant in Toronto, Canada. The experiments were performed under diverse operational conditions using $250 \mathrm{~mL}$ serum bottles at room temperature $\left(22 \sim 25^{\circ} \mathrm{C}\right)$, neutral $\mathrm{pH}(6.5$ $\sim 7.5$ ), and a shaking speed of $165 \mathrm{rpm}$. The reaction liquid medium volume was equal to $50 \mathrm{~mL}$, whereas, methane and oxygen were added to the headspace after being evacuated as described in AlSayed et al. (2017). The model was built using 49 data sets with 10 inputs (listed in Table 1) and two outputs (described in section 2.2). These 49 data sets were collected throughout 12 different batch experiments (performed in triplicates) which are described in detail in AlSayed et al. $(2017,2019)$ and AlSayed et al. (2018b). Over the experiments, varied nitrogen, copper, methane, and oxygen concentrations, initial biomass densities, food to microorganisms $(F / M)$, nitrogen to microorganisms $(N / M)$, carbon to nitrogen $(C / N)$, and oxygen to methane $(O / M)$ ratios were applied (the ranges are shown in Table 1). All the incubations were completed within $24 \pm 4$ hours. Along with the duration, final biomass density $\left(B_{f}\right)$ and final methane concentration $\left(M_{f}\right)$ were measured to determine microbial growth rate $\left(h r^{-1}\right)$, methane growth yield $(g-D C W / g$ $\left.\mathrm{CH}_{4 \text { consumed }}\right)$, and methane uptake rate $\left(\mathrm{mg}-\mathrm{CH}_{4} / \mathrm{hr}\right)$ to assess the methanotrophic microbial activity.

Table 1. Process Input Parameters for ANN Models

\begin{tabular}{lll}
\hline Parameter & Unit & Range \\
\hline Core factors & & \\
Methane volume & $\mathrm{mL}$ & $25 \sim 200$ \\
Oxygen volume & $\mathrm{mL}$ & $25 \sim 200$ \\
Nitrogen concentration & $\mathrm{mM}$ & $10 \sim 160$ \\
Copper concentration & $\mu \mathrm{M}$ & $0 \sim 80$ \\
Initial biomass density & $\mathrm{OD} 600$ & $0.2 \sim 3$ \\
Ratio factors & & \\
$F / M$ ratio & $\mathrm{g}-\mathrm{CH} / \mathrm{g}-\mathrm{DCW}$ & $1.3 \sim 20.7$ \\
$C / N$ ratio & $\mathrm{gC}-\mathrm{CH} 4 / \mathrm{g}-\mathrm{N}$ & $0.25 \sim 14$ \\
$N / M$ ratio & $\mathrm{g}-\mathrm{N} / \mathrm{g}-\mathrm{DCW}$ & $0.5 \sim 8.5$ \\
$O / M$ ratio & $\mathrm{mL} / \mathrm{mL}$ & $1 \sim 4$ \\
\hline
\end{tabular}

\subsection{ANN Model Development}

A series of MLP ANN models comprising one input layer, one hidden layer and one output layer was used in this research to predict both the final biomass density $\left(B_{f}\right)$ and final methane concentration $\left(M_{f}\right)$ of enriched methanotrophic bacteria. The selection of input parameters for each configuration is detailed in section 2.3, while the selection of the optimum ANN architecture is detailed in section 2.4. The two output parameters $\left(B_{f}\right.$ and $M_{f}$ ) are the controlling variables that govern the KBM coefficients and were selected as the model outputs since they are the direct outcome measured from the laboratory experiments. The KBM coefficients can be determined according to Equations 5 to 7 , given that time is constant and equal to 20 hours on average:

$$
\begin{aligned}
& \text { Microbial growth rate, } \mu=\frac{\frac{B_{f}-B_{i}}{\text { time }}}{\frac{B_{f}+B_{i}}{2}} \\
& \text { Methane growth yield, } Y=\frac{B_{f}-B_{i}}{M_{f}-M_{i}} \\
& \text { Methane uptake rate, } M U R=\frac{M_{f}-M_{i}}{\text { time }}
\end{aligned}
$$

where $B_{i}$ and $M_{i}$ are the initial biomass density, and initial methane concentration, respectively.

All model data (input and output data) were pre-processed before training the ANN by normalizing each dataset between the range $[0,1]$. This step is an important process and governs overall ANN performance since it ensures that all the variables get an equal attention. A variety of the training (i.e., calibrating) algorithms exist to develop ANNs including but not limited to Levenberg-Marquardt, Bayesian Regularization and Scaled Conjugate Gradient logarithms (Tu, 1996). The LevEnberg-Marquardt $(L M)$ backpropagation algorithm was selected as the training algorithm as it is considered as one of the most efficient algorithms for ANN models (Ahmadian, 2016). This is a gradient descent algorithm that adjusts the weights and biases of the ANN to minimize the error between the calculated outputs and the target outputs. The $L M$ algorithm was selected due to its ability to use a hybrid technique which combines both GaussNewton and steepest descent approaches to find the potential optimal solution. The previous technique is highly suitable in the case of models that have large number of parameters with nonlinear relationship which makes it a good fit for the models used in this study (Lourakis, 2005; Wilson and Mantooth, 2013). Prior to ANN model architecture optimization, the full dataset was randomly split into a 70\%:15\%:15\% ratio for training, validation and testing datasets, respectively. An early-stopping algorithm was used to terminate the training once the mean squared error of the validation samples starts to increase, indicating that model generalization has reduced (Alvisi et al., 2006). Only the testing (i.e., independent) dataset was 
used for model performance and inference purposes. The network was trained using both 5 and 10 neurons in the hidden layer $\left(N_{H}\right)$ for comparison purposes. Further refinements into the size of the hidden layer was conducted during the optimization phase (detailed in section 2.4).

Each model was trained 100 times (known as multi-start) to account for the random initialization used for the training algorithm. By retraining the model several times, it ensures that the uncertainty in model calibration and the variability in each model performance is accounted for. A collection of these 100 repeated trained models for the same input, output and architecture configuration is referred to as an ensemble.

The normalized mean square error (NRMSE) and the squared Pearson correlation coefficient $\left(R^{2}\right)$ for each case were calculated using Equations (8) and (9) and compared to assess the model performance. The NRMSE (rather than RMSE) was used as the values for both outputs $\left(B_{f}\right.$ and $M_{f}$ ) have different range - the normalization allows for a direct comparison across different model configurations and ensembles:

$$
\begin{aligned}
& R^{2}=\left(\frac{\operatorname{cov}\left(Y_{o b s}, Y_{m o d}\right)}{\left(\sigma_{o b s} \sigma_{\text {mod }}\right)}\right)^{2} \\
& \text { NRMSE }=\left(\frac{R M S E}{\text { mean }\left(Y_{o b s}\right)}\right)
\end{aligned}
$$

where $Y_{o b s}$ and $Y_{m o d}$ are the observed and predicted values for each output variable, RMSE is the root mean squared error between the $Y_{o b s}$ and $Y_{m o d}$.

\subsection{ANN Input Variable Selection}

The selection of inputs for an ANN model are a significant source of uncertainty in overall model performance and are the key factors that contribute to a successful ANN model (Khan et al., 2018). Thus, the selection of inputs to predict both $B_{f}$ and $M_{f}$ a two-step procedure was used. First, six model ensembles were created consisting of five "core" factors and four "ratio" factors (as listed in Table 1). Methanotrophic microbial activity is influenced by multiple operational and environmental conditions (Semrau et al., 2010). The most influential factors are the methane and oxygen concentrations (two of the core factors). Methane is the sole source that provide the cells with its energy requirements and cellular carbon representing about $50 \%$ of the cellular dry weight. Whereas, oxygen is the electron acceptor which is needed with satisfactory concentrations to maintain the aerobic respiration of methane (Hanson and Hanson, 1996; Madigan et al., 2015). Therefore, no methanotrophic activity is expected to take place in the absence of methane and lor oxygen.

Furthermore, nitrogen concentration, added as sodium nitrate, has been shown to notably affect the methanotrophic kinetics as one of the macro-nutrients essential for any microbial growth. However, it was recently reported that excessive nitrate addition may inhibit the microbial activity due to the associated increased salinity (AlSayed et al., 2019). Copper pres- ence is a decisive parameter that directly controls the expression of the MMO enzyme responsible of catalyzing methane oxidation, and by consequence, all the metabolic activities by the cells (Semrau et al., 2010). Methane diffusion from the gaseous phase to the liquid phase is one of the main limitations to model methanotrophs growth kinetics. It is directly related to the methanotrophic biomass density in the experiments (Stone et al., 2017). Thus, nitrogen, copper and initial biomass density were included in the set of candidate inputs as the remaining "core" factors. Collectively, it can be concluded that methane, oxygen, nitrogen, copper, and biomass concentrations are the five core factors that needs to be considered in modelling the microbial activity of any methanotrophic culture.

In addition to the core factors, four "ratio" factors have been reported to affect the microbial activity independent from the effect of the core factors. These four ratio factors are $O / M$, $F / M, N / M$, and $C / N$ (Tchobanoglous et al., 2003; AlSayed et al., 2017). The ratio factors are correlated and dependent on the core factors which may negatively affect the model performance. Thus, in this research the impact of the "ratio" factors on the final $B_{f}$ and $M_{f}$ concentration is explored. First, a control model is developed that includes all nine candidate inputs: five core and four ratio factors. Following this, in order to enhance the model performance, the model was retrained after eliminating $F / M, C / N, N / M$, and $O / M$ ratios from the inputs, sequentially. Lastly, a model that only includes the five core factors was developed. Comparing these six model configurations will help determine the influence of the ratio factors on the final $B_{f}$ and $M_{f}$ concentrations, as well as the ability of ANN models to implicitly account for their physical influence (since the ratios are a function of the core factors).

Subsequently, in order to minimize the large number of inputs (initially nine inputs in total), IVS was carried out to the model ensembles to eliminate the input variables that have minor to no impact on the output values or ANN performance. This step may enhance the model performance by eliminating the irrelevant variables resulting in a less complex and more accurate model. Moreover, this step would help investigating the impact of the different operational parameters on the outputs towards a better understanding of the process a key objective of this research. First, the network was trained using the simplest model consisting of only methane and oxygen concentrations as inputs and $B_{f}$ and $M_{f}$ as outputs. Subsequently, each one of the remaining input variables (nitrogen and copper concentrations and initial biomass density, representing the operational parameter) were added separately to the previous two inputs variables (methane and oxygen concentration).

Thus, to meet this objective, eight ensemble ANN models were trained using different combinations of the core factors. One model includes all nine input variables, and one includes only methane and oxygen. Three models included methane, oxygen and one each of the operational parameters (initial biomass, copper, and nitrogen), and three models that included methane, oxygen and two of the operational parameters (nitrogen and copper, nitrogen and initial biomass, and initial biomass and copper). The performance of the eight ensemble models was quantified using NRMSE and $R^{2}$. 


\subsection{ANN Architecture Optimization and Uncertainty Analysis}

Two main factors in ANN architecture that contribute to the uncertainty in model performance are the number of nodes in the hidden layers $\left(N_{H}\right)$ and the data division (amount of data used for training, validation, and testing). Choosing a well-balanced number of nodes is crucial towards developing a successful model since having fewer than the required $N_{H}$ might be not sufficient to enable a good representation of the system. On the other hand, having a higher $N_{H}$ can result in overfitting meaning that model has lost its ability to generalize (Maier and Dandy, 2000). Similarly, identifying the amount of data used for training, validation, and testing is a key factor as using a small amount of data for training can result in poor model performance and using more data will result in less data set for validating and testing (which may be insufficient for model inference). Typically, both $N_{H}$ and amount of data used for data division are selected based on a trial and error method, however a coupled method to identify the optimum $N_{H}$ and data division has been proposed (Khan and Valeo, 2017b). In this research, the previously suggested method has been followed with some minor adjustments to fit our model and is described below.

To start the network architecture optimization, the data division was randomly selected as 70\%:15\%:15\% for training, validation and testing, respectively. The network was then trained using 5 inputs (the core factors only) using different number of nodes in the hidden layer (specifically $2,4,6,8,10,12,14$, 16,18 , and 20 neurons) to identify the optimum $N_{H}$ for the model. This step was repeated 100 times for each $N_{H}$ to take in consideration the variation in the model performance for the same iteration. Following this, the process was repeated using the optimum number of neurons selected in the previous step but using different amount of data for training $(10,20,30,40,50,60,70$, and $80 \%)$ and each iteration was run 100 times as well. The NRMSE and $R^{2}$ were calculated for each step and the number of nodes and percentage of data division that led to the lowest $N R M S E$ and highest $R^{2}$ were selected as the optimum $N_{H}$ and the optimum data division.

\section{Results and Discussions}

\subsection{Influence of Ratio Factors on ANN Performance}

Six ANN ensemble models were trained using different combinations of the core and ratio factors, using both 5 and 10 $N_{H}$ for each configuration. Figure 3 shows the median values for $R^{2}$ and NRMSE for the ensemble models for the two outputs $M_{f}$ and $B_{f}$. As shown in Figure 3, no notable difference can be observed between the models with all nine inputs and the models without the ratio factors. In contrast, a minor improvement is observed by removing all the ratio factors. Additionally, no clear difference in the pattern of model performance can be observed when $N_{H}$ varied from 5 to 10 . A recent study has investigated the significance of $F / M, N / M$, and $C / N$ ratio: the results illustrated that $F / M$ has significant effect on the methanotrophic microbial activity, while, $N / M$ and $C / N$ has limited or no effect on methanotrophs growth (AlSayed et al., 2017). In contrast, the results from the models imply that the model has as- signed all ratio factors minimal weights, and by consequence, minor effect on the prediction of both outputs. It can be deduced from these results that the ANN models have considered the effect of the ratios implicitly (since they are derived from the core factors) without a need to include them as explicit input parameters. This is a significant result that highlights the utility of the ANN approach for modelling methanotrophic processes.

In $\mathrm{KBM}$, the ratio factors must be considered apart from the core factors which add more sophistications in the model (Tchobanoglous et al., 2003). Moreover, parameters like N/M and $C / N$ ratio may have been overlooked or neglected if their effect has not been quantified or studied in the literature. Nonetheless, it was shown in this section that the effect of those ratios is already considered in the model which is demonstrated by the good performance of the model. This is the reason why it is proposed that DDM is superior tool to model emerging technology. Simply put, processes can be modelled and designed prior to being fully understood and physically/chemically/ biologically quantified. As an example, the effect of N/M ratio on methanotrophs was not reported until recently (AlSayed et al., 2017) and has not been modelled yet. Here, it is worth mentioning that the inhibition mechanisms of ammonium (as nitrogen) on methanotrophic microorganisms is significantly differrent than other microbes. This is due to its unique possession of the MMO enzyme and its similarities with the AMO enzyme responsible for ammonium oxidation (AlSayed et al., 2018a). As a result, more studies are still needed to be able to quantify it and model it. Yet, such effect was already considered in the DDM without even the need to include it as independent variable or consider it as a variable.

Therefore, ratio factors were eliminated to avoid the correlation between the input variables and consequently minimize redundancy resulting in a final ANN model configuration with 5 core factors as the inputs and the 2 outputs. Such a finding emphasizes the advantage for DDM over KBM as it can build indirect and complex relationships between the inputs and outputs without being fully physically or mathematically understood.

\subsection{Influence of Operational Variables on ANN Performance}

Another advantage for the constructed ANN model is its capacity to assess each of the input variables effect on the outputs. Eight model configurations were developed to investigate the influence of the core factors on the methanotrophic microbial activity. As mentioned above, methane and oxygen are crucial for methanotrophic growth and no activity is expected in their absence. So, methane and oxygen have been selected as the minimum inputs to develop the simplest model with the fewest inputs. Figure 4 shows the modelled and target values for the $B_{f}$ and $M_{f}$ for the training, validation, and testing for the configuration where only methane and oxygen are used as inputs. It can be concluded that the model failed to appropriately describe the outputs. For example, the model with $N_{H}=10$, the median $R^{2}$ values are 0.197 and 0.175 , whereas, the median $N R M S E$ values are 0.759 and 0.521 for $B_{f}$ and $M_{f}$, respectively. 

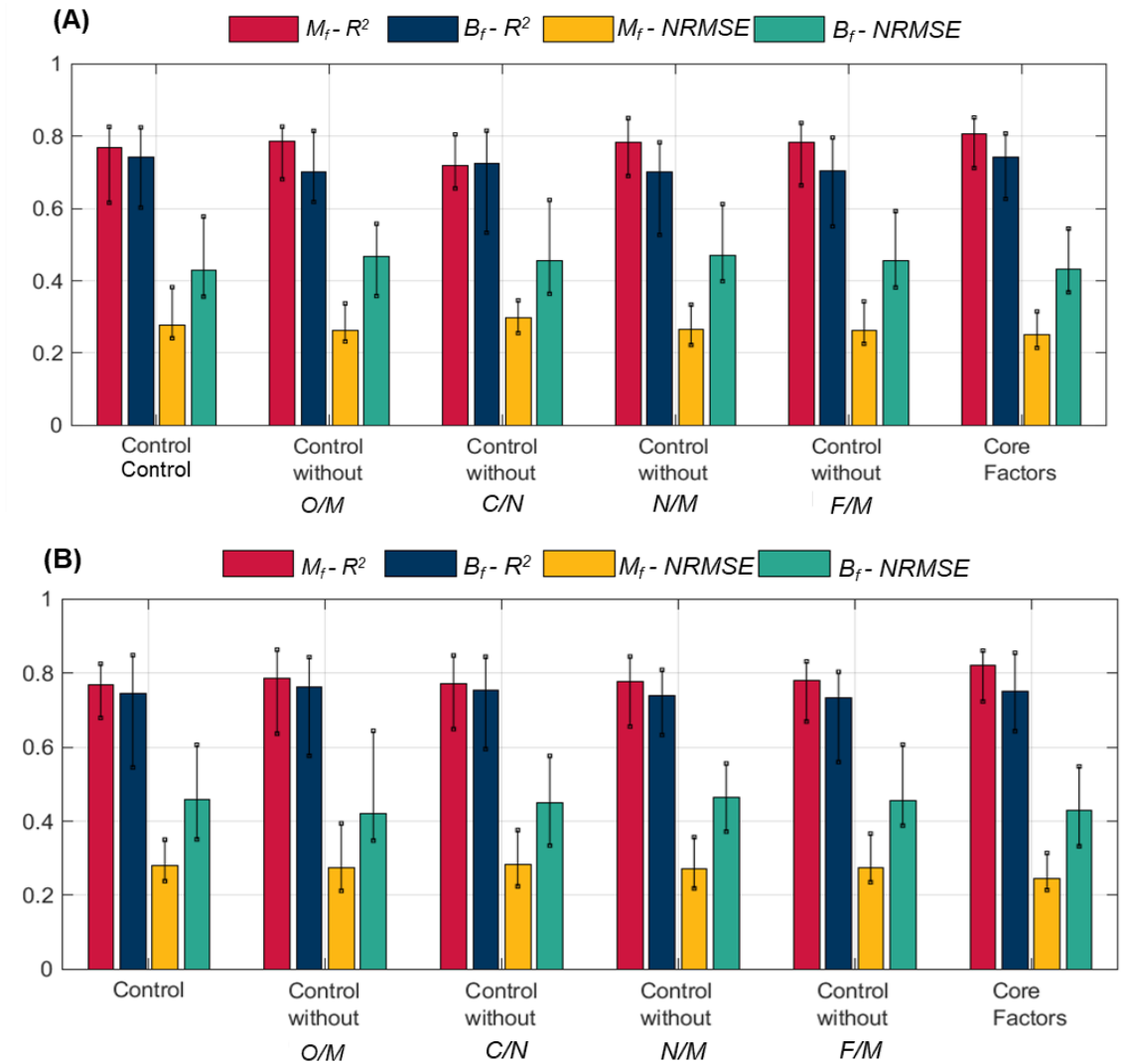

Figure 3. The effect of ratio factors on the model performance. Presented values are for the median and error bars show the lower and upper quartiles, (A) $N_{H}=5$ and (B) $N_{H}=10$.
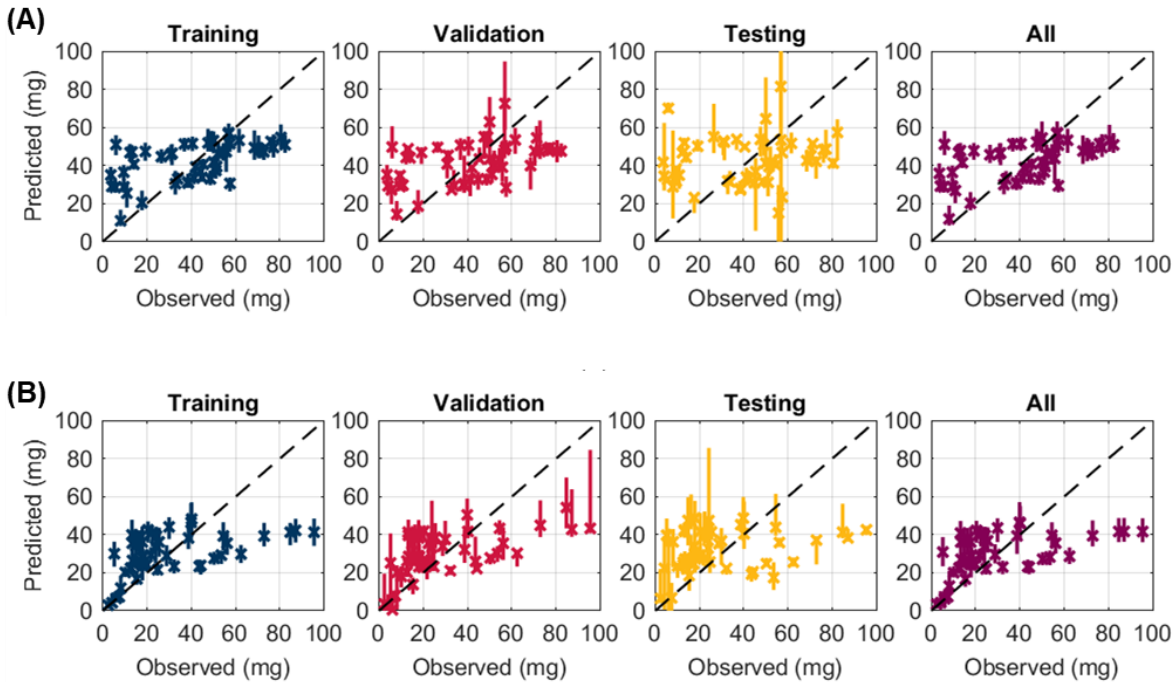

Figure 4. Correlation between modelled and measured outputs using methane and oxygen as the only inputs, (A) for $B_{f}$ and (B) for $M_{f}$.

Commonly, KBM relies on the electron acceptor/donor concentration to predict microbial activity (Rittmann and McCarty, 2012). Showing poor performance when only using KBM inputs can be used to highlight that KBM is not accurate enough to describe and predict the microbial activity as seen in the data through the laboratory experiments. Furthermore, it confirms the merit of this research of including the other operational factors as key inputs to describe the microbial activity using DDM.

Subsequently, two additional sets of models were developed: three models that included methane, oxygen and one of 
the operational factors (initial biomass, copper, and nitrogen), and three models that included methane, oxygen and combinations of two of the other operation factors (nitrogen and copper, nitrogen and initial biomass, and initial biomass and copper). The objective of these models is to better understand the influence of copper concentration, nitrogen concentration, and initial biomass density on the culture activity. Furthermore, it is an attempt to examine the model capacity to rank the influence of each factor on the outputs. Figure 5 presents the median values of $R^{2}$ and $N R M S E$ values for $B_{f}$ and $M_{f}$.

The presence of copper is crucial for methane uptake since it is a micronutrient that is needed by methanotrophs to maintain the MMO activity (Semrau et al., 2010). The variation in copper concentration did not dramatically affect either the methanotrophic growth or the methane uptake (AlSayed et al., 2019). Moreover, it is usually added with trace concentrations range from 5 to $20 \mu M$ (Bowman, 2006). This phenomenon is demonstrated in Figure 5 where it is apparent that copper is the least influential parameter on the $M_{f}$ and $B_{f}$, since it has the lowest in $R^{2}$ and NRMSE for both outputs while using $N_{H}$ values of 5 and 10 .

On the other hand, the addition of initial biomass density or the nitrogen concentration resulted in similar improvements on model performance, as shown in Figure 5. This observation shows that the model has assigned similar weights for both factors and that similar attention should be paid to both parameters to enhance the microbial activity in an operational setting. High initial biomass density hinders the attainment of higher methanotrophic activity. It affects the methane diffusivity into the liquid phase from the gaseous phase which results in limited microbial activity (Han et al., 2009). Moreover, it is directly related to the $F / M$ ratio that was recently reported to notably affect the methanotrophic activity (AlSayed et al., 2017). The other parameter is nitrogen which is widely reported that increaseing nitrogen concertation when added in the form of nitrate has notable influence on the microbial activity (AlSayed et al., 2019). This influence can be deduced by the fact that nitrogen represents up to $13 \%$ of the cellular materials (Madigan et al., 2015). Moreover, some recent studies reported that some methanotrophic strains can utilize nitrate as the electron acceptor to secure the needed energy requirements under oxygen limited conditions (Alrashed et al., 2018). Combining these facts together, it can be concluded that nitrogen is crucial for methanotrophs to support its requirements of energy and cell synthesis requirements.

Lastly, similar findings can be seen when methane and oxygen are combined with two other inputs (copper, nitrogen, and initial biomass concentration) as shown in Figure 5. In these models (each of which have four inputs in total), the model performance (expressed as NRMSE and $R^{2}$ ) was higher than those models that only three inputs (methane, oxygen and one of the operational parameters) described above. Moreover, models developed using nitrogen concentration and initial biomass density is clearly better than the other two models based on the performance metrics. Whereas, the elimination of either initial biomass density or nitrogen concentration resulted in similar performance. These observations agree with two findings discuss- ed above: first, that the inclusion of more independent operational and environmental parameters results in better prediction of the microbial activity. This finding is supported by the improvement attained when the five core factors are used as the input variables. Secondly, nitrogen concentration and initial biomass density have almost the same ranking in terms of effect on the microbial activity, while, copper has a lower influence.

\subsection{ANN Architecture Optimization}

The aim of this analysis was to identify the optimum $N_{H}$ and data division for the ANN configuration - focusing specially on the models with the five core factors as inputs. First, the amount of data was randomly split into 70, 15 and $15 \%$ ratio for training, validation and testing, respectively and the model was trained using different values for $N_{H}$, and repeated 100 times to take in consideration the random selection of the data. The median, the quartile, the upper and lower limits for each number of neurons are shown in Figure 6. The figure shows that increasing the number of neurons from 2 to 8 resulted in a slight decrease in the NRMSE and an increase in $R^{2}$ for the trained data for both outputs. It is noteworthy that increasing the $N_{H}$ to 8 resulted in a lower variation in the quartile values for both $B_{f}$ and $M_{f}$. In terms of validation and testing, the NRMSE and $R^{2}$ values were not considerably affected by changing the number of neurons. On the other hand, further increase in number of neurons (from 8 to 20) did not result in notable improvement for the NRMSE and $R^{2}$ for $B_{f}$ (as seen in Figure 6(A) and (B) and instead an increase in the quartile values is evident. Similarly, increasing the $N_{H}$ from 8 to 20 for the model with $M_{f}$ resulted in limited improvement of the model performance, even though that the $R^{2}$ values for the training data increased. This increase was accompanied with an increase in the model variability as shown in Figure 6(C) and (D). The previous results imply that using a simpler model may be more adequate to describe the model and reduce computational effort. Hence, $N_{H}$ of 8 was selected as the optimum number of neurons in the hidden layer.

Following this, the influence of the data division on the model performance was investigated using the optimum number of neurons $\left(N_{H}=8\right)$. As shown in Figure $7(\mathrm{~A})$ and $(\mathrm{C})$, increasing the percentage of trained data from 20 to $80 \%$ resulted in notable decrease in the NRMSE of the validation and testing and no notable change in the training NRMSE for both the model outputs $\left(B_{f}\right.$ and $\left.M_{f}\right)$ implying an improvement in the model performance. Similarly, the $R^{2}$ values followed a similar trend to that of the NRMSE values as shown in Figure 7(B) and (D). The previous results revealed that the model required more training data to be better represent the measured data and that increasing the training percentage to $80 \%$ did not result in overfitting to the training data since the remaining data set was still sufficient for a robust statistical inference of the data set. Therefore, it can be concluded that using 80,10 , and $10 \%$ for training, validation, and testing, respectively, is the optimum data division for this dataset.

To assess the impact of including different operational conditions such as copper and nitrogen concentrations and 

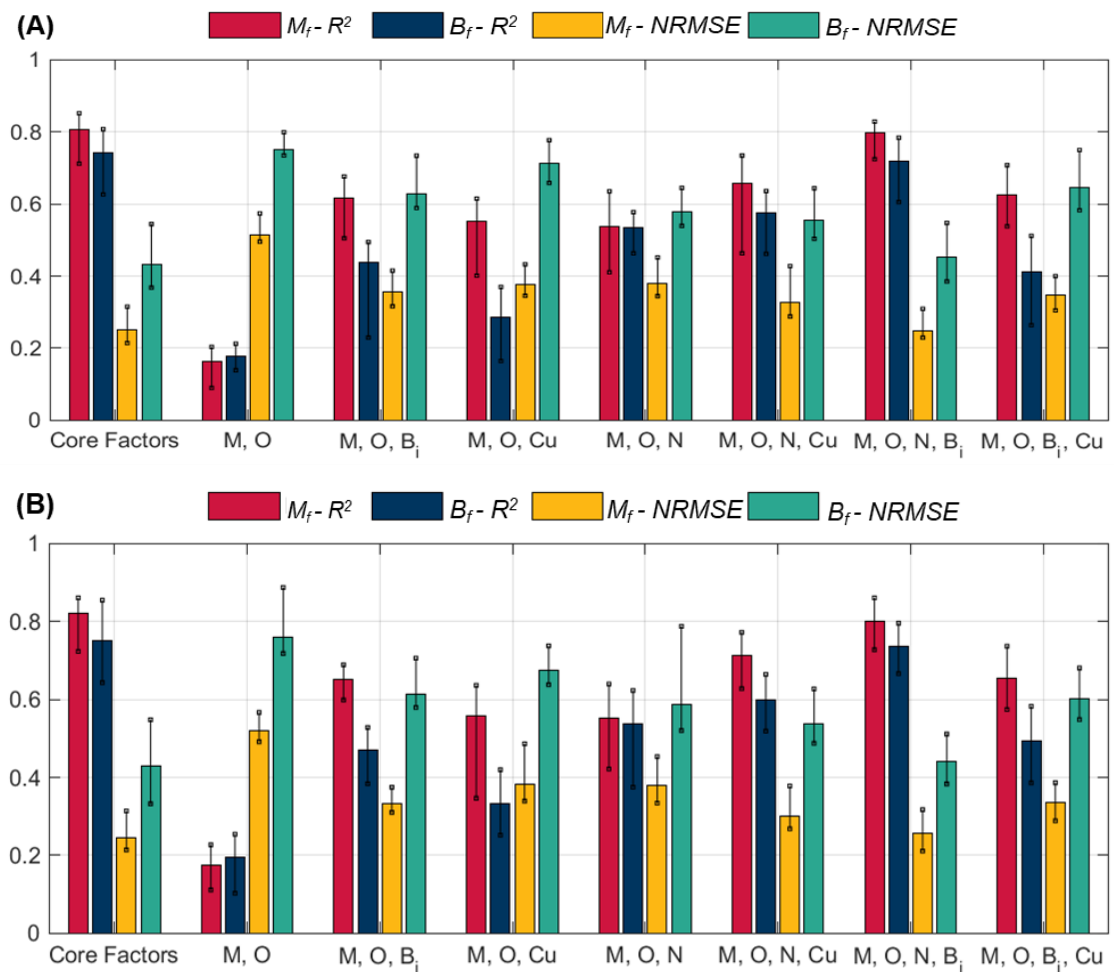

Figure 5. Model performance with different combinations of input variables. Presented values are for the median and error bars show the lower and upper quartiles, (A) $N_{H}=5$, (B) $N_{H}=10$.

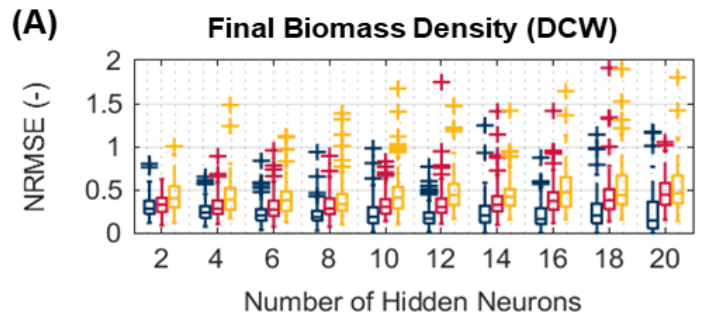

(B) Final Biomass Density (DCW)

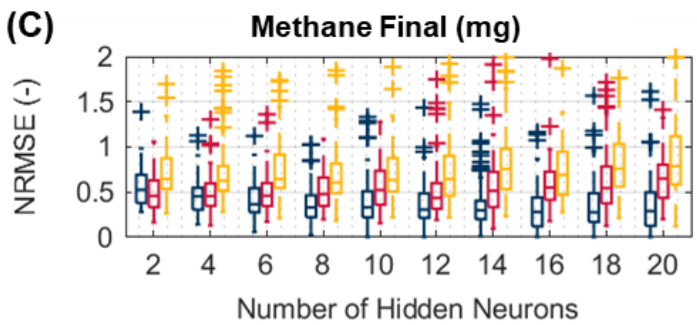

(D)
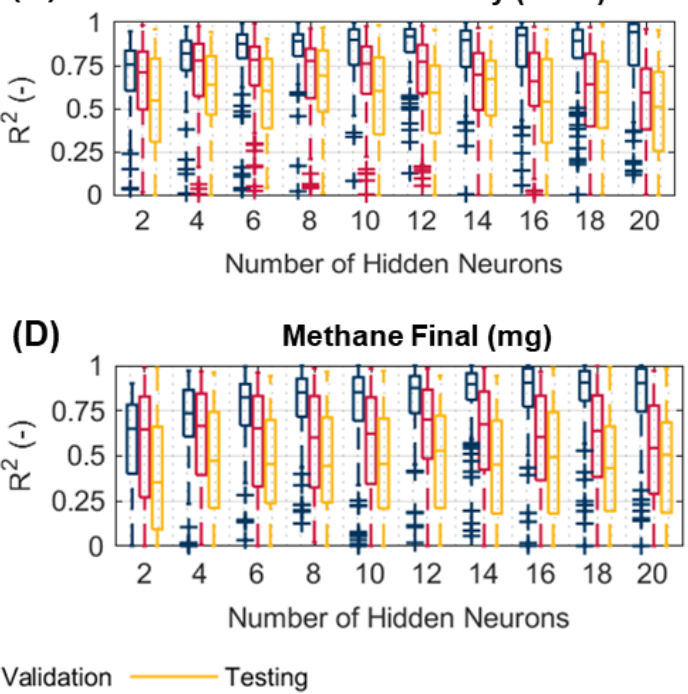

Figure 6. Number of Neurons $\left(\mathrm{N}_{\mathrm{H}}\right)$ influence on the outputs of the model with 5 variable inputs using data split ratio of 70, 15, and $15 \%$ for training, validation and testing, respectively: (A) NRMSE for $B_{f},(\mathrm{~B}) R^{2}$ for $B_{f}$, (C) NRMSE for $M_{f}$, and (D) $R^{2}$ for $M_{f}$.

initial biomass density as input variables on the model performance the previous optimization was repeated using a model with only two inputs: methane and oxygen concentration only (rather than the five core factors). As illustrated in Figures 8 and 9, relying only on the electron donor and acceptor (KBM inputs) to model the microbial activity of the methanotrophic bacteria resulted in a poor model performance using all differrent combinations of $N_{H}$ and data division which confirms the findings of this research that the other core factors (i.e., the operational factors) are important contributors and predictors of methanotrophic processes. This data should be included in any models attempting to describe the modelling behavior, and data 
driven models, such as ANNs are able to incorporate the differrent operational conditions resulting in more accurate models than typical KBM.

\subsection{Final ANN Model Performance}

After ANN model architecture determination, the model was retrained using the optimum data division (80\%:10\%:10\%) and optimum $N_{H}(8)$ and the results of the model median values for NRMSE and $R^{2}$ are presented in Table 2 . The measured and modelled data values for the $B_{f}$ and $M_{f}$ for the training, validation, testing, and all data, were plotted for the final model (Figure 10). As illustrated, the final model was able to accurately predict the measured outputs at all the different operational conditions implying the success of the ANN in simulating the cultivation of mixed culture methanotrophs type I bacteria enriched from sewage sludge using multiple environmental conditions.

Table 2. Overall Model Performance under Optimum Parameters

\begin{tabular}{lllll}
\hline & Output & & \\
\cline { 2 - 5 } & $B_{f}$ & & \\
\cline { 2 - 5 } & $N R M S E$ & $R^{2}$ & $N R M S E$ & $R^{2}$ \\
\hline Training & 0.2 & 0.88 & 0.33 & 0.85 \\
Validation & 0.25 & 0.82 & 0.41 & 0.75 \\
Testing & 0.35 & 0.7 & 0.59 & 0.58 \\
All & 0.23 & 0.84 & 0.39 & 0.79 \\
\hline
\end{tabular}

\subsection{Significance and Implications}

An MLP ANN model to simulate the cultivation of methanotrophs type I bacteria enriched from sewage sludge under different conditions has been developed. In order to overcome the challenges encountering the KBM, a data driven approach has been followed taking in consideration all the factors that might have an effect on the culture performance. These factors included methane, oxygen, nitrogen, copper, and biomass concentrations which were grouped as the core factors, as well as four ratio factors which are $O / M, F / M, N / M$, and $C / N$. During the model construction phase, it was revealed that eliminating the ratio factors did not affect the model performance, rather a slight improvement was observed. This finding emphasizes the benefits of using DDM in identifying the relations between inputs and outputs that have not been fully understood yet either mathematically or physically. Moreover, the impact of the copper, nitrogen and initial biomass concentrations on the output parameters has been investigated using 8 different models comprising of different combinations of the core factors. The results showed that the nitrogen and initial biomass concentrations are more influential on the microbial activity than the copper concentration, which is in accordance to what have been reported in the literature. In addition, it was revealed that incurporating more environmental and operational factors as the model inputs resulted in a better performance than that of using only the methane and oxygen as the only model inputs. The previous results consolidate the hypothesis of favoring the data driven approach over the physical models for simulating the microbial growth, as the latter does not account for the change in other operational conditions (i.e., when only methane and oxygen concentration is considered). Furthermore, in order to optimize the network architecture, a previously proposed protocol has been followed with some minor adjustments to select the optimum number of neurons on the hidden layer $\left(N_{H}\right)$ as well as the optimum amount of data for training, validation, and testing (Khan and Valeo, 2017b). The results demonstrated that using a $N_{H}=8$ and data division of 80,10 , and $10 \%$ for training, validation, and testing, respectively, results in the best model performance.
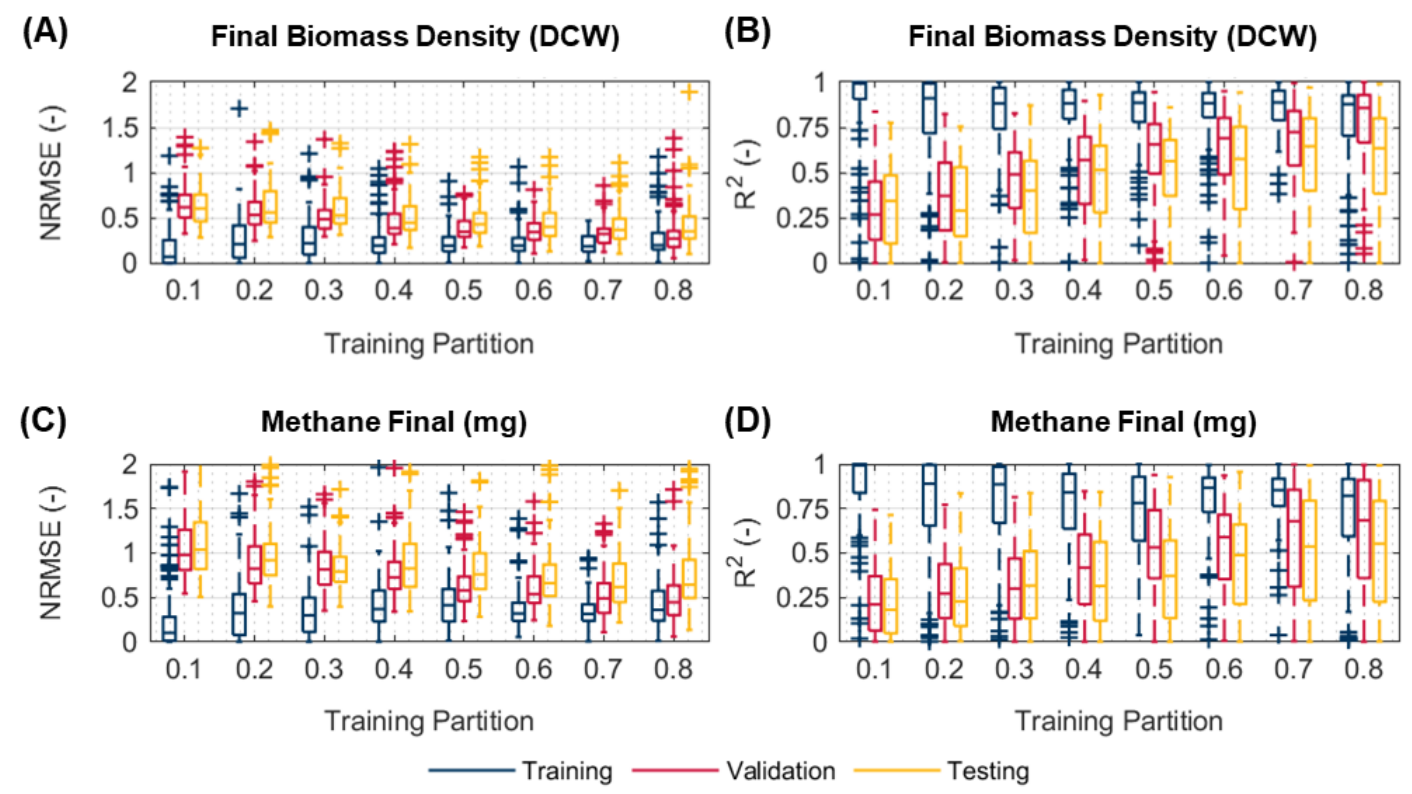

Figure 7. Percentage of trained data influence on the performance of the model with 5 input variables using $N_{H}=8:(\mathrm{A}) N R M S E$ for $B_{f}$, (B) $R^{2}$ for $B_{f}$, (C) $N R M S E$ for $M_{f}$, and (D) $R^{2}$ for $M_{f}$. 
(A)

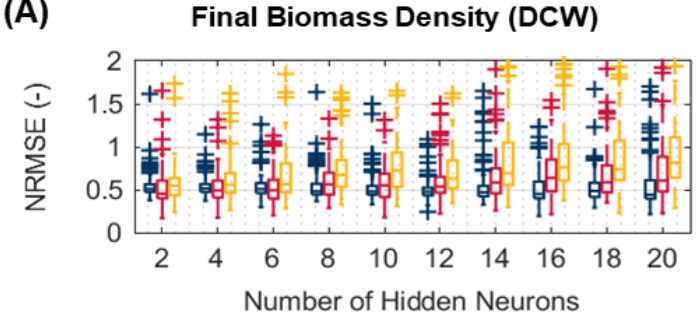

(C)

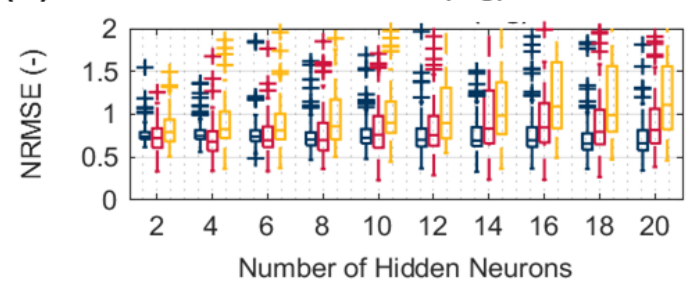

(B) Final Biomass Density (DCW)

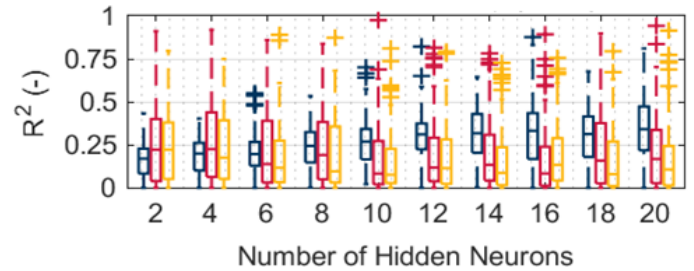

(D)

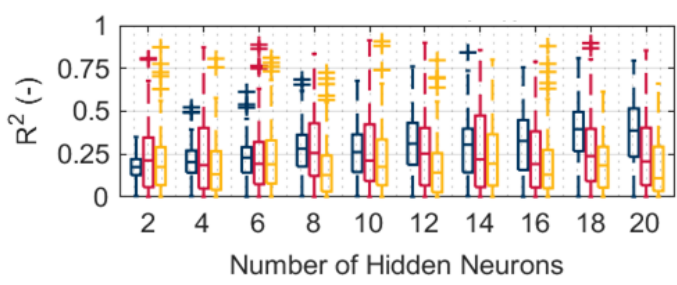

Training Validation Testing

Figure 8. Number of Neurons $\left(N_{H}\right)$ influence on the outputs of the model with 2 variable inputs using data split ratio of 70,15 , and $15 \%$ for training, validation and testing, respectively: (A) $N R M S E$ for $B_{f}$, (B) $R^{2}$ for $B_{f}$, (C) $N R M S E$ for $M_{f}$, and (D) $R^{2}$ for $M_{f}$.

(A) Final Biomass Density (DCW)

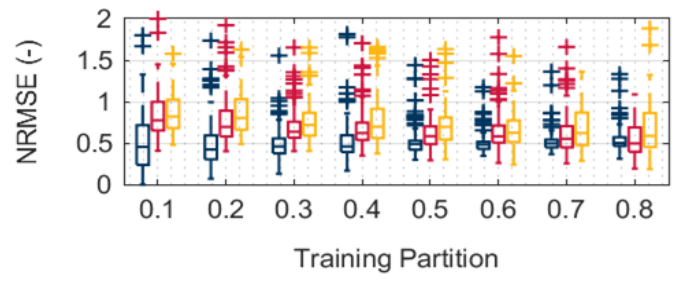

(C)

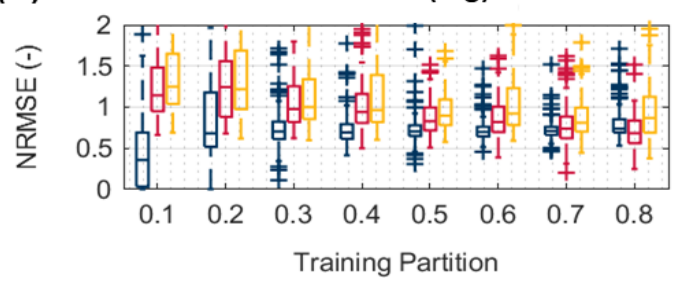

(B) Final Biomass Density (DCW)

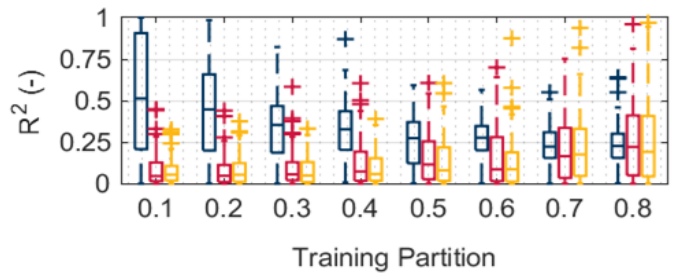

(D)

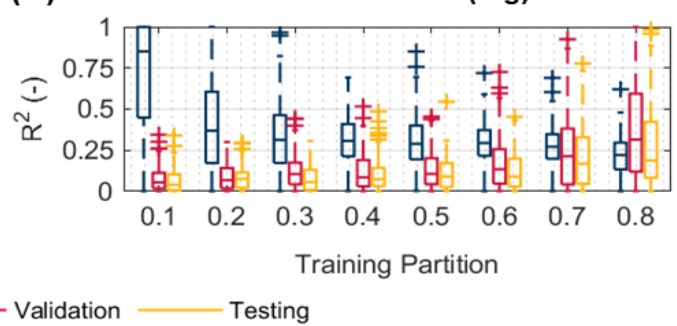

Figure 9. Percentage of trained data influence on the performance of the model with 2 input variables using $N_{H}=8$ : (A) $N R M S E$ for $B_{f}$, (B) $R^{2}$ for $B_{f}$, (C) NRMSE for $M_{f}$, and (D) $R^{2}$ for $M_{f}$.

Currently, there is a push to substitute the well-understood and matured process in STPs with more energy and cost-efficient processes. Nonetheless, such alternative/emerging processes are complex and not fully understood which challenge the shift from STPs to WRRFs. This study proposes the employment of DDM models to model the emerging systems as an alternative to KBM. Given its multiple biotechnological applications, methanotrophic-based bioprocesses are strong candidate to be utilized in WRRFs to revalorize the typically flared biogas in STPs. Therefore, this study developed an ANN modelling approach to simulate the enrichment of a mixed culture type I methanotrophs. The success of the proposed modelling approach demonstrates a proof-of-concept of the potential to use of DDM models in designing WRRFs. The complexity of methanotrophs-based bioprocesses can be owing to its sensitivity to different environmental conditions such as copper and nitrogen concentrations. In additions, the process includes the interaction of three phases: gaseous (methane and oxygen), aqueous (the nutrients medium and the metabolic intermediates), and solid (the mixed microbial consortium). As a result, significant variation in the reported KBM output is evident as demonstrated in Figure 1.

In this study, the ability of the ANN model to consider the complex relationships between the inputs without the need to add them separately as individual inputs was demonstrated. In contrast, in the KBM, the relationship between the operational 


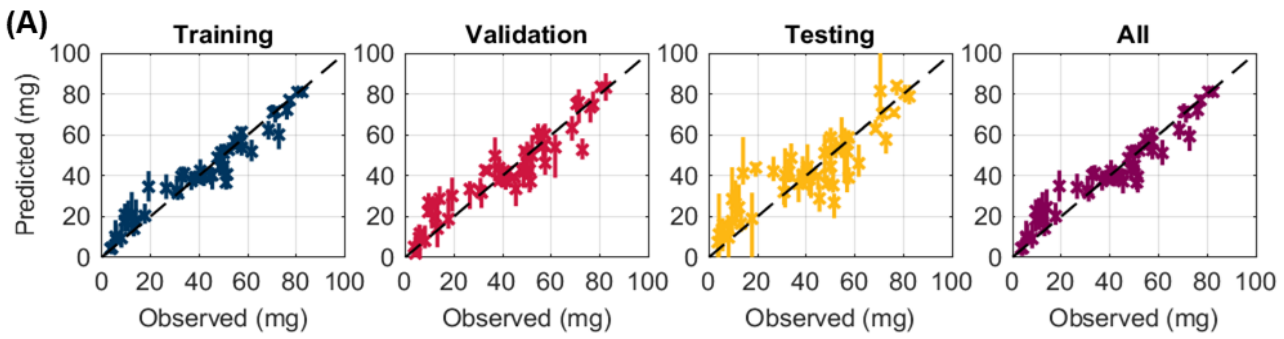

(B)
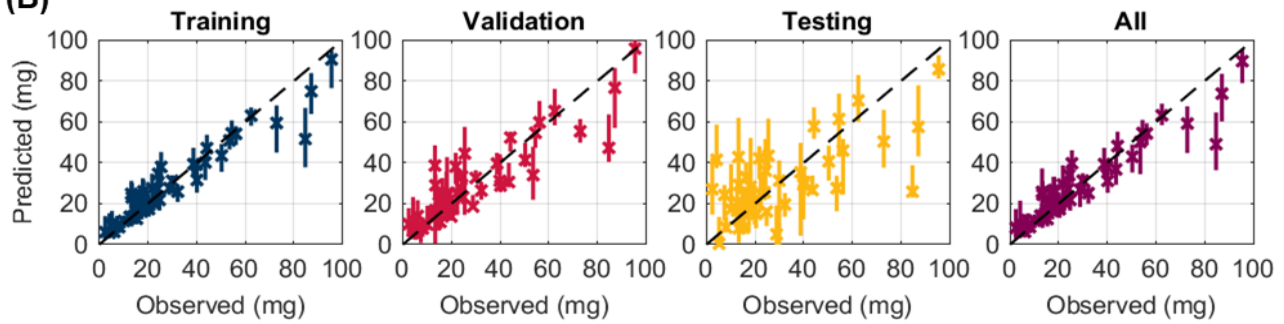

Figure 10. Correlation between modelled and measured outputs of the final model: (A) for $B_{f}$ and (B) for $M_{f}$.

parameters must be included. Thus, it can be implied that, using DDM using data obtained from conditions mimicking the actual conditions, novel bioprocesses can be validated prior of being fully understand and considering all the factors affecting it. In addition, the model found to be able to assess the effect of different operational conditions and rank these operational conditions according to their significance effect on the outcome. These findings address one of the drawbacks of the KBM of relying only on the electron donor and acceptor to predict the microbial activity. In addition, the study highlighted the significance of: (i) optimizing the number of neurons in the hidden layer $\left(N_{H}\right)$ and (ii) data division in order to generate an accurate ensemble of models to stimulate the cul- tivation of the targeted bioprocess. Collectively, the findings of this research pave the way for using DDM to model different biological processes in order to facilitate the transition from the current STPs operation to the WRRF concept.

\section{Conclusion}

Over the last decade, the shift towards WRRFs is gaining traction due to several environmental and economic benefits. The emerging bioprocesses in WRRFs are not fully understood from the microbiological and kinetic perspectives leading to difficulties in using kinetics-based models. Thus, the emergence of new technologies to support WRRFs is hampered by the lack of models to support the design of bioreactors and to better understand the bioprrocesses. In this research, data driven models (DDM) are proposed as a robust alternative towards modelling emerging bioprocesses. DDM utilization for modelling the biological processes is a prominent area of research that can boost the adoption of new bioprocesses that support the shift STPs to WRRFs. A proof-of-concept that DDM has the potential to replace KBM to more accurately describe and predict the microbial activity in WRRFs is proposed in this research. It is the first study to model the overall process of methanotrophs, which can be extended to other microbes and bioprocesses. This research demonstrated that ANN models can be used to better understand and rank the influence of different input variables (i.e., the physical parameters that influence methanotrophs) on the microbial activity. Further investigation can be performed to estimate the model limitations to clarify to what extent can data driven models accurately describe microbial cultures. In addition, modelling commercial scale reactors of bioprocesses would include much more input variables and expose higher model complexity which would require more advanced IVS technique.

Acknowledgements. The authors would like to express their appreciation to three anonymous reviewers for their constructive comments which improved the quality of their paper. They would also like to thank the Natural Sciences and Engineering Research Council (NSERC) of Canada for its financial support.

\section{References}

Ahmadian, A.S. (2016). Chapter 6 Numerical Methods and Procedures. Numerical Models for Submerged Breakwaters, 93-108. https://doi.org/ 10.1016/B978-0-12-802413-3.00006-7

Alrashed, W., Lee, J., Park, J., Rittmann, B.E., Tang, Y., Neufeld, J.D., and Lee, H.S. (2018). Hypoxic methane oxidation coupled to denitrification in a membrane biofilm. Int. J. Chem. Eng., 348, 745-753. https://doi.org/ 10.1016/j.cej.2018.04.202

AlSayed, A., Fergala, A., and Eldyasti, A. (2017). Influence of biomass density and food to microorganisms ratio on the mixed culture type I methanotrophs enriched from activated sludge. J. Environ. Sci., 70, 87-96. https://doi.org/10.1016/j.jes.2017.11.017

AlSayed, A., Fergala, A., and Eldyasti, A. (2018a). Sustainable biogas mitigation and value-added resources recovery using methanotrophs intergrated into wastewater treatment plants. Rev. Environ. Sci. Bio., 1-43. https://doi.org/10.1007/s11157-018-9464-3

AlSayed, A., Fergala, A., Khattab, S., and Eldyasti, A. (2018b). Kinetics of type I methanotrophs mixed culture enriched from waste activated sludge. Biochem. Eng. J., 132, 60-67. https://doi.org/10.1016/j.bej. 2018.01.003

AlSayed, A., Fergala, A., and Eldyasti, A. (2019). Enhancement of the 
cultivation process conditions of mixed culture methanotrophic Proteobacteria phylum enriched from waste activated sludge as the first step for value added recovery process. J. Biosci. Bioeng., 127(5), 602-608. https://doi.org/10.1016/j.jbiosc.2018.10.018

Asztalos, J.R. and Kim, Y. (2017). Lab-scale experiment and model study on enhanced digestion of wastewater sludge using bioelectrochemical systems. J. Environ. Inform., 29(2), 98-109.

Boiesen, A., Arvin, E., and Broholm, K. (1993). Effect of mineral nutrients on the kinetics of methane utilization by methanotrophs. Biodegradation, 4(3), 163-170. https://doi.org/10.1007/BF00695118

Bowman, J. (2006). The Methanotrophs - The Families Methylococcaceae and Methylocystaceae. In The Prokaryotes (fourth, Vol. 5). Springer New York.

Canadian Biogas Association. (2013). Canadian Biogas Study: Benefits to the Economy, Environment and Energy.

Chen, Y., Cheng, J.J., and Creamer, K.S. (2008). Inhibition of anaerobic digestion process: A review. Bioresour. Technol., 99(10), 4044-4064. https://doi.org/10.1016/j.biortech.2007.01.057

Delhoménie, M.-C., Nikiema, J., Bibeau, L., and Heitz, M. (2008). A new method to determine the microbial kinetic parameters in biological air filters. Chem. Eng. Sci., 63(16), 4126-4134. https://doi.org/10. 1016/j.ces.2008.05.020

Fergala, A., AlSayed, A., and Eldyasti, A. (2018a). Factors affecting the selection of PHB accumulating methanotrophs from waste activated sludge while utilizing ammonium as their nitrogen source. $J$. Chem. Technol. Biotechnol., 93(5), 1359-1369. https://doi.org/10. $1002 /$ jctb.5502

Fergala, A., AlSayed, A., and Eldyasti, A. (2018b). Behavior of type II methanotrophic bacteria enriched from activated sludge process while utilizing ammonium as a nitrogen source. Int. Biodeterior. Biodegradation, 130, 8-16. https://doi.org/10.1016/j.ibiod.2018.03.010

Foresti, E., Zaiat, M., and Vallero, M. (2006). Anaerobic Processes as the Core Technology for Sustainable Domestic Wastewater Treatment: Consolidated Applications, New Trends, Perspectives, and Challenges. Rev. Environ. Sci. Bio., 5(1), 3-19. https://doi.org/10.1007/ s11157-005-4630-9

Ge, X., Yang, L., Sheets, J.P., Yu, Z., and Li, Y. (2014). Biological conversion of methane to liquid fuels: Status and opportunities. Biotechnol. Adv., 32(8), 1460-1475. https://doi.org/10.1016j.biotechadv. 2014.09.004

Govindaraju, R.S. (2000). Artificial Neural Networks in Hydrology. I: Preliminary Concepts. J. Hydrol. Eng., 5(2), 115-123. https://doi.org/ 10.1061/(ASCE)1084-0699(2000)5:2(115)

Gu, Y., Li, Y., Li, X., Luo, P., Wang, H., Wang, X., Wu, J., and Li, F. (2017). Energy Self-sufficient Wastewater Treatment Plants: Feasibilities and Challenges. Energy Procedia, 105(Supplement C), 3741-3751. https://doi.org/10.1016/j.egypro.2017.03.868

Han, B., Su, T., Wu, H., Gou, Z., Xing, X.H., Jiang, H., Chen, Y., Li, X., and Murrell, J.C. (2009). Paraffin oil as a "methane vector" for rapid and high cell density cultivation of Methylosinus trichosporium OB3b. Appl. Microbiol. Biotechnol., 83(4), 669-677. https://doi.org/10.1007/ s00253009-1866-2

Hanson, R.S. and Hanson, T.E. (1996). Methanotrophic bacteria. Microbiol. Rev., 60(2), 439-471. https://doi.org/10.1128/MMBR.60.2.439-471. 1996

Heijnen, J.J. and Roels, J.A. (1981). A macroscopic model describing yield and maintenance relationships in aerobic fermentation processes. Biotechnol. Bioeng., 23(4), 739-763. https://doi.org/10.1002/bit. 260230407

Jimenez, J., Miller, M., Bott, C., Murthy, S., De Clippeleir, H., and Wett, B. (2015). High-rate activated sludge system for carbon management Evaluation of crucial process mechanisms and design parameters. Water Res., 87, 476-482. https://doi.org/10.1016/j.watres.2015.07.032

Khan, U.T., He, J., and Valeo, C. (2018). River flood prediction using fuzzy neural networks: an investigation on automated network architecture. Water Sci. and Technol., 2017(1), 238-247. https://doi.org/10.2166/ wst. 2018.107

Khan, U.T. and Valeo, C. (2016a). Short-Term Peak Flow Rate Prediction and Flood Risk Assessment Using Fuzzy Linear Regression. $J$. Environ. Inform, 28(2). https://doi.org/10.3808/jei.201600345

Khan, U.T. and Valeo, C. (2016b). Dissolved oxygen prediction using a possibility theory based fuzzy neural network. Hydrol. Earth Syst. Sci., 20(6), 2267, https://doi.org/10.5194/hess-20-2267-2016.

Khan, U.T. and Valeo, C. (2017a). Comparing A Bayesian and Fuzzy Number Approach to Uncertainty Quantification in Short-Term Dissolved Oxygen Prediction. J. Environ. Inform., 30(1). https://doi. org/10.3808/jei.201700371

Khan, U.T. and Valeo, C. (2017b). Optimising Fuzzy Neural Network Architecture for Dissolved Oxygen Prediction and Risk Analysis. Water, 9(6), 381. https://doi.org/10.3390/w9060381

Kisi, O., Akbari, N., Sanatipour, M., Hashemi, A., Teimourzadeh, K., and Shiri, J. (2013). Modeling of Dissolved Oxygen in River Water Using Artificial Intelligence Techniques. J. Environ. Inform., 22(2). https://doi. org/10.3808/jei.201300248

Liao, J.C., Mi, L., Pontrelli, S., and Luo, S. (2016). Fuelling the future: microbial engineering for the production of sustainable biofuels. Nat. Rev. Microbiol., 14(5), 288-304. https://doi.org/10.1038/nrmicro. 2016.32

Lourakis, M.I. (2005). A brief description of the Levenberg-Marquardt algorithm implemented by levmar. Fou. Rese. and Technol., 4(1), 1-6

Madigan, M.T., Martinko, J.M., Bender, K.S., Buckley, D.H., and Stahl, D.A. (2015). Brock biology of microorganisms. Pearson (14th ed.).

Maier, H.R. and Dandy, G.C. (2000). Neural networks for the prediction and forecasting of water resources variables: a review of mod- elling issues and applications. Environ. Modell. Softw., 15(1), 101- 124. https://doi.org/10.1016/S1364-8152(99)00007-9

McCarty, P.L., Bae, J., and Kim, J. (2011). Domestic Wastewater Treatment as a Net Energy Producer-Can This be Achieved? Environ. Sci. Technol., 45(17), 7100-7106. https://doi.org/10.1021/es2014264

Ménard, C., Ramirez, A.A., and Heitz, M. (2014). Kinetics of simultaneous methane and toluene biofiltration in an inert packed bed. J. Chem. Technol. Biotechnol., 89(4), 597-602. https://doi.org/10.1002/jctb.4162

Nourani, V., Baghanam, A.H., and Gebremichael, M. (2012). Investigating the Ability of Artificial Neural Network (ANN) Models to Estimate Missing Rain-gauge Data. J. Environ. Inform., 19(1). https://doi.org/ 10.3808/jei.201200207

Ordaz, A., López, J.C., Figueroa-González, I., Muñoz, R., and Quijano, G. (2014). Assessment of methane biodegradation kinetics in two-phase partitioning bioreactors by pulse respirometry. Water Res., 67, 46-54. https://doi.org/10.1016/j.watres.2014.08.054.

Puyol, D., Batstone, D.J., Hülsen, T., Astals, S., Peces, M., and Krömer, J.O. (2017). Resource Recovery from Wastewater by Biological Technologies: Opportunities, Challenges, and Prospects. Front. Microbiol., 7. https://doi.org/10.3389/fmicb.2016.02106

Remesan, R., Bray, M., and Mathew, J. (2018). Application of PCA and clustering methods in input selection of hybrid runoff models. $J$. Environ. Inform, 31(2), 137-152. https://doi.org/10.3808/jei.201700378

Rittmann, B.E. and McCarty, P.L. (2012). Environmental biotechnology: principles and applications. Tata McGraw-Hill Education.

Rostkowski, K.H., Pfluger, A.R., and Criddle, C.S. (2013). Stoichiometry and kinetics of the PHB-producing Type II methanotrophs Methylosinus trichosporium OB3b and Methylocystis parvus OBBP. Bioresour. Technol., 132, 71-77. https://doi.org/10.1016/j.biortech.2012.12.129

Sancho, I., Lopez-Palau, S., Arespacochaga, N., and Cortina, J.L. (2019). New concepts on carbon redirection in wastewater treatment plants: A review. Sci. Total. Environ., 647, 1373-1384. https://doi.org/10.1016/j. scitotenv.2018.08.070

Semrau, J.D., DiSpirito, A.A., and Yoon, S. (2010). Methanotrophs and copper. FEMS Microbiol. Rev., 34(4), 496-531. https://doi.org/10.1111/ j.1574-6976.2010.00212.x

Siniscalchi, L.A.B., Vale, I.C., Dell'Isola, J., Chernicharo, C.A., and Calabria Araujo, J. (2015). Enrichment and activity of methanotrophic 
microorganisms from municipal wastewater sludge. Environ. Technol., 36(12), 1563-1575. https://doi.org/10.1080/09593330.2014.997298

Soliman, M. and Eldyasti, A. (2017). Long-term dynamic and pseudostate modeling of complete partial nitrification process at high nitrogen loading rates in a sequential batch reactor (SBR). Bioresour. Technol., 233, 382-390. https://doi.org/10.1016/j.biortech.2017.02.108

Solomatine, D.P. and Ostfeld, A. (2008). Data-driven modelling: some past experiences and new approaches. J. Hydroinform., 10(1), 3-22. https:// doi.org/10.2166/hydro.2008.015

Snieder, E., hakir, R., and Khan, U.T. (2019). A comprehensive comparison of four input variable selection methods for artificial neural network flow forecasting models. J. Hydrol., 583, 124299, https://doi. org/10.1016/j.jhydrol.2019.124299.

Stone, K. A., Hilliard, M.V., He, Q.P., and Wang, J. (2017). A mini review on bioreactor configurations and gas transfer enhancements for biochemical methane conversion. Biochem. Eng. J., 128, 83-92. https:// doi.org/10.1016/j.bej.2017.09.003

Tchobanoglous, G., Burton, F.L., and Stensel, H.D. (2003). Wastewater Engineering: Treatment and Reuse. McGraw-Hill (4th ed.).

Tu, J.V. (1996). Advantages and disadvantages of using artificial neural networks versus logistic regression for predicting medical outcomes. $J$. Clin. Epidemiol., 49(11), 1225-1231. https://doi.org/10.1016/S08954356(96)00002-9

US EPA, (2016). Fast Facts from the Inventory of U.S. Greenhouse Gas Emissions and Sinks: 1990-2014 [Overviews and Factsheets]. https://www.epa.gov/ghgemissions/fast-facts-inventory-usgreenhouse-gas-emissions-and-sinks-1990-2014

Wan, J., Gu, J., Zhao, Q., and Liu, Y. (2016). COD capture: a feasible option towards energy self-sufficient domestic wastewater treatment. Sci. Rep., 6. https://doi.org/10.1038/srep25054

Wilson, P. and Mantooth, H.A. (2013). Model-based engineering for complex electronic systems. Newnes. 